\title{
Trade-off in antibody escape and ACE2 binding of the SARS-Cov2 spike protein generates new variants which should ideally be lesser efficient, transmissible and pathogenic
}

\section{Letter}

A recent spate of reports of variants of concern (VOC) of SARS-Cov2 have arisen in different countries [1, 2] (https://www.cdc.gov/coronavirus/2019-ncov/cases-updates/variant-surveillance/variant-info.html). While, such mutations will undoubtedly escape previous antibodies $[3,4]$, these are also constrained by the SARSCov2 spike protein's (SCSP) interaction with the ACE2 protein [5]. Thus, these variants may turn out to be lesser efficient in cell-entry and thus replication. The fast declining cases South Africa after the detection of variants suggest this might be true. Furthermore, antibodies to other viral proteins and cell-mediated immunity (T-cells) also provide protection against these variants.

\section{Comprehensive set of antibodies binding to the SARS-Cov2 spike protein}

Here, I analyze known antibodies with solved structures in the PDB database. Most antibodies bind to the RBD:receptor-binding domain, barring one which binds to the HR1: heptapeptide repeat sequence 1 (912-984). $14(50 \%)$ of these make contact to one of the three mutations (K417/E484/N501) in the SA variant. It is most likely that antibodies would have lesser efficiency against such variants. However, these would come at a cost to the virus - since these variants would also need to bind the ACE2 receptor.

\section{Residues in SARS-Cov2 spike protein involved in ACE2 binding:}

Among the residues involved directly in ACE2 binding, N501 is prominent - the slight difference in binding caused by the N501Y mutation is empirically seen in Table 2. The residues of ACE2 involved in the interaction with the SCSP - especially the +ve Lys353 and the -ve Asp355 - indicates the constraints faced by SCSP while mutating, since the charge distribution in that region cant be changed. None of the other variants of concern till date (B.1.1.7 (UK), P.1 (Brazil), B.1.351 (SA), https://www.cdc.gov/coronavirus/2019ncov/cases-updates/variant-surveillance/variant-info.html) are involved in ACE2 binding directly. Some of the antibodies that make contact to the VOC - and may thus be rendered less effective in neutralizing viral entry is shown in Table 3.

\section{A small primer on the SARS-Cov2 spike protein}

The spike protein is fragmented [6] into the following subunits S1 (14-685 residues) and S2 (686-1273), subdivided into: NTD:N-terminal domain (14-305 residues), RBD:receptor-binding domain (RBD, 319-541 residues), FP: fusion peptide (788-806), HR1: heptapeptide repeat sequence 1 (912-984), HR2: heptapeptide repeat sequence 2 (1163-1213), TM: transmembrane domain (1213-1237), CT: cytoplasm domain $(1237-1273)$

\section{References}

1. Burki T (2021) Understanding variants of SARS-Cov-2. The Lancet 397: 462.

2. Tang JW, Tambyah PA, Hui DS (2020) Emergence of a new SARS-Cov-2 variant in the UK. The Journal of Infection .

3. Wang P, Lihong L, Iketani S, Luo Y, Guo Y, et al. (2021) Increased resistance of SARS-Cov-2 variants B.1.351 and B.1.1.7 to antibody neutralization. BioRxiv . 


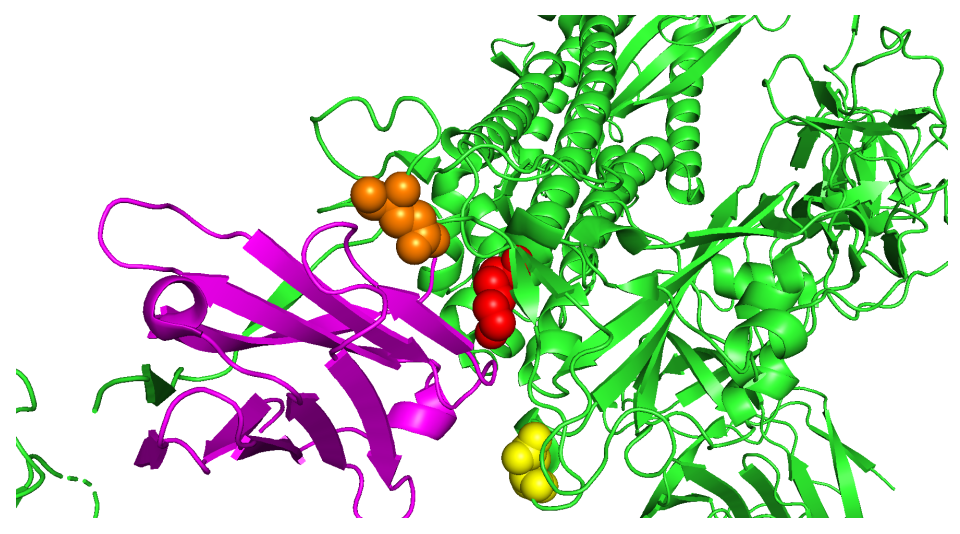

Figure 1: Spike protein (green) with bound antibody (magenta): Making contacts to all three variants of concern (B.1.1.7 (UK), P.1 (Brazil), B.1.351 (SA)) mutations in RBD (E484 in orange, N501 in yellow and K417 in red). PDBid:7JWB.

4. Kuzmina A, Khalaila Y, Voloshin O, Keren-Naus A, Bohehm L, et al. SARS-Cov-2 escape variants exhibit differential infectivity and neutralization sensitivity to convalescent or post-vaccination sera. Available at SSRN 3789258 .

5. Dong J, Huang B, Jia Z, Wang B, Gallolu Kankanamalage S, et al. (2020) Development of multispecific humanized llama antibodies blocking SARS-Cov-2/ACE2 interaction with high affinity and avidity. Emerging microbes \& infections 9: 1034-1036.

6. Huang Y, Yang C, Xu Xf, Xu W, Liu Sw (2020) Structural and functional properties of SARS-Cov-2 spike protein: potential antivirus drug development for COVID-19. Acta Pharmacologica Sinica 41: $1141-1149$.

7. Yuan M, Wu NC, Zhu X, Lee CCD, So RT, et al. (2020) A highly conserved cryptic epitope in the receptor binding domains of sars-cov-2 and sars-cov. Science 368: 630-633.

8. Li D, Edwards RJ, Manne K, Martinez DR, Schäfer A, et al. (2021) The functions of sars-cov-2 neutralizing and infection-enhancing antibodies in vitro and in mice and nonhuman primates. bioRxiv : 2020-12.

9. Piccoli L, Park YJ, Tortorici MA, Czudnochowski N, Walls AC, et al. (2020) Mapping neutralizing and immunodominant sites on the sars-cov-2 spike receptor-binding domain by structure-guided highresolution serology. Cell 183: 1024-1042.

10. Wrobel AG, Benton DJ, Hussain S, Harvey R, Martin SR, et al. (2020) Antibody-mediated disruption of the sars-cov-2 spike glycoprotein. Nature communications 11: 1-5.

11. Zhou D, Duyvesteyn HM, Chen CP, Huang CG, Chen TH, et al. (2020) Structural basis for the neutralization of sars-cov-2 by an antibody from a convalescent patient. Nature structural \& molecular biology 27: 950-958.

12. Yuan M, Liu H, Wu NC, Lee CCD, Zhu X, et al. (2020) Structural basis of a shared antibody response to sars-cov-2. Science 369: 1119-1123.

13. Hansen J, Baum A, Pascal KE, Russo V, Giordano S, et al. (2020) Studies in humanized mice and convalescent humans yield a sars-cov-2 antibody cocktail. Science 369: 1010-1014. 
14. Hurlburt NK, Seydoux E, Wan YH, Edara VV, Stuart AB, et al. (2020) Structural basis for potent neutralization of sars-cov-2 and role of antibody affinity maturation. Nature communications 11: 1-7.

15. Kreye J, Reincke SM, Kornau HC, Sánchez-Sendin E, Corman VM, et al. (2020) A therapeutic nonself-reactive sars-cov-2 antibody protects from lung pathology in a covid-19 hamster model. Cell 183: $1058-1069$.

16. Lv Z, Deng YQ, Ye Q, Cao L, Sun CY, et al. (2020) Structural basis for neutralization of sars-cov-2 and sars-cov by a potent therapeutic antibody. Science 369: 1505-1509.

17. Custódio TF, Das H, Sheward DJ, Hanke L, Pazicky S, et al. (2020) Selection, biophysical and structural analysis of synthetic nanobodies that effectively neutralize sars-cov-2. Nature communications 11: $1-11$.

18. Bertoglio F, Fühner V, Ruschig M, Heine PA, Rand U, et al. (2020) A sars-cov-2 neutralizing antibody selected from covid-19 patients by phage display is binding to the ace2-rbd interface and is tolerant to known rbd mutations .

19. Ju B, Zhang Q, Ge J, Wang R, Sun J, et al. (2020) Human neutralizing antibodies elicited by sars-cov-2 infection. Nature 584: 115-119.

20. Cao Y, Su B, Guo X, Sun W, Deng Y, et al. (2020) Potent neutralizing antibodies against sars-cov-2 identified by high-throughput single-cell sequencing of convalescent patients' b cells. Cell 182: 73-84.

21. Du S, Cao Y, Zhu Q, Yu P, Qi F, et al. (2020) Structurally resolved sars-cov-2 antibody shows high efficacy in severely infected hamsters and provides a potent cocktail pairing strategy. Cell 183: 10131023.

22. Shi R, Shan C, Duan X, Chen Z, Liu P, et al. (2020) A human neutralizing antibody targets the receptor-binding site of SARS-Cov-2. Nature 584: 120-124.

23. Bracken CJ, Lim SA, Solomon P, Rettko NJ, Nguyen DP, et al. (2021) Bi-paratopic and multivalent vh domains block ace2 binding and neutralize sars-cov-2. Nature Chemical Biology 17: 113-121.

24. Clark SA, Clark LE, Pan J, Coscia A, McKay LG, et al. (2020) Molecular basis for a germline-biased neutralizing antibody response to sars-cov-2. bioRxiv .

25. Rujas E, Kucharska I, Tan YZ, Benlekbir S, Cui H, et al. (2020) Multivalency transforms sars-cov-2 antibodies into broad and ultrapotent neutralizers. bioRxiv .

26. Barnes CO, Jette CA, Abernathy ME, Dam KMA, Esswein SR, et al. (2020) Sars-cov-2 neutralizing antibody structures inform therapeutic strategies. Nature : 1-6.

27. Wu NC, Yuan M, Liu H, Lee CCD, Zhu X, et al. (2020) An alternative binding mode of ighv3-53 antibodies to the sars-cov-2 receptor binding domain. Cell reports 33: 108274.

28. Wec AZ, Bornholdt ZA, He S, Herbert AS, Goodwin E, et al. (2019) Development of a human antibody cocktail that deploys multiple functions to confer pan-ebolavirus protection. Cell host \& microbe 25 : $39-48$.

29. Acharya P, Williams W, Henderson R, Janowska K, Manne K, et al. (2020) A glycan cluster on the sars-cov-2 spike ectodomain is recognized by fab-dimerized glycan-reactive antibodies. bioRxiv .

30. Zhou T, Tsybovsky Y, Gorman J, Rapp M, Cerutti G, et al. (2020) Cryo-em structures of sars-cov2 spike without and with ace 2 reveal a ph-dependent switch to mediate endosomal positioning of receptor-binding domains. Cell host \& microbe 28: 867-879. 
Table 1: Almost complete set of antibodies $(\mathbf{N}=\mathbf{2 7})$ in the PDB database tiil date: Most antibodies bind to the RBD:receptorbinding domain (RBD, 319-541 residues), barring one which binds to the HR1: heptapeptide repeat sequence 1 (912-984). 14 of these (6XC4A 6XE1H 7B3OE 7C8WA 7CDJE 7CHEH 7CJFA 7CWOA 7JMPA 7JWBD 7K9ZH 7KFWA 7KN6A) make contact to one of the three mutations (K417/E484/N501) in the SA varant. The residue on the spike protein which is closest to the antibody is given, along with the distance.

\begin{tabular}{|c|c|c|c|c|}
\hline PDB & Spike & Antibody & $\AA$ & Paper - description \\
\hline 7CJF & $\mathrm{ASP} / 420 / \mathrm{OD} 2$ & SER/56/OG & 2.4 & not published \\
\hline $6 \mathrm{~W} 41$ & GLY/381/O & TYR/32/OH & 2.2 & "A highly conserved cryptic epitope in the RBD " [7] \\
\hline 7LD1 & TYR/369/O & TYR/128/OH & 2.6 & "..neutralizing and infection-enhancing antibodies in vitro and in mice and nonhuman primates" [8] \\
\hline $7 \mathrm{JVC}$ & $\mathrm{PHE} / 377 / \mathrm{N}$ & SER/31/O & 3.0 & "Mapping Neutralizing and Immunodominant Sites on the Spike RBD" [9] \\
\hline 7A5S & $\mathrm{SER} / 383 / \mathrm{OG}$ & THR/104/OG1 & 2.7 & Antibody-mediated disruption of the spike glycoprotein. [10] \\
\hline $6 \mathrm{ZFO}$ & THR/385/OG1 & TYR/106/OH & 2.3 & "Structural basis for the neutralization by an antibody from a convalescent patient." [11] \\
\hline $6 \mathrm{XC} 4$ & $\mathrm{ASP} / 420 / \mathrm{OD} 2$ & $\mathrm{SER} / 56 / \mathrm{OG}$ & 2.6 & "Structural basis of a shared antibody response to ." [12] \\
\hline 6XDG & $\mathrm{PHE} / 486 / \mathrm{CE} 1$ & $\mathrm{ASP} / 92 / \mathrm{O}$ & 3.2 & "Studies in humanized mice and convalescent humans yield a antibody cocktail." [13] \\
\hline 6XE1 & LEU/455/O & $\mathrm{TYR} / 33 / \mathrm{OH}$ & 2.6 & Structural basis for potent neutralization ... and role of antibody affinity maturation. [14]. \\
\hline $6 \mathrm{XKP}$ & TYR/449/N & $\mathrm{SER} / 98 / \mathrm{O}$ & 2.7 & "Therapeutic Non-self-reactive .. Antibody Protects from Lung Pathology in a Hamster Model." [15] \\
\hline 7CAI & $\mathrm{PHE} / 374 / \mathrm{O}$ & $\mathrm{PHE} / 92 / \mathrm{N}$ & 2.5 & "Structural basis for neutralization by a potent therapeutic antibody." [16]. \\
\hline $7 \mathrm{~A} 29$ & $\mathrm{ASN} / 450 / \mathrm{OD} 1$ & $\mathrm{HIS} / 35 / \mathrm{NE} 2$ & 2.8 & Selection, biophysical and structural analysis of synthetic nanobodies that effectively neutralize . [17] \\
\hline 7B3O & $\mathrm{ASP} / 420 / \mathrm{OD} 2$ & $\mathrm{SER} / 56 / \mathrm{OG}$ & 2.4 & "selected ...by phage display ...ACE2-RBD interface .. tolerant to known RBD mutations [18]. \\
\hline 7BWJ & GLY/447/O & $\mathrm{TYR} / 27 / \mathrm{OH}$ & 2.9 & "Human neutralizing antibodies elicited by infection. [19]. \\
\hline 7BYR & $\mathrm{TYR} / 449 / \mathrm{OH}$ & $\mathrm{LEU} / 72 / \mathrm{O}$ & 2.7 & Potent Neutralizing Antibodies against Identified ...of Convalescent Patients' B Cells." [20] \\
\hline $7 \mathrm{CHE}$ & $\mathrm{LEU} / 455 / \mathrm{O}$ & $\mathrm{TYR} / 33 / \mathrm{OH}$ & 2.4 & "..Shows High Efficacy in Severely Infected Hamsters and Provides a Potent Cocktail Pairing Strategy" [21] \\
\hline 7C01 & $\mathrm{ASP} / 420 / \mathrm{OD} 2$ & $\mathrm{SER} / 56 / \mathrm{OG}$ & 2.5 & "A human neutralizing antibody targets the RBD site " [22] \\
\hline 7JWB & $\mathrm{TYR} / 505 / \mathrm{OH}$ & $\mathrm{SER} / 31 / \mathrm{O}$ & 2.7 & "Bi-paratopic and multivalent VH domains block ACE2 binding and neutralize ." [23] \\
\hline $7 \mathrm{KFW}$ & $\mathrm{TYR} / 453 / \mathrm{OH}$ & GLY $/ 99 / O$ & 2.5 & "Molecular basis for a germline-biased neutralizing antibody response to ." [24] \\
\hline $7 \mathrm{KN} 6$ & $\mathrm{TYR} / 473 / \mathrm{OH}$ & $\mathrm{SER} / 31 / \mathrm{O}$ & 2.4 & unpublished \\
\hline $7 \mathrm{~K} 9 \mathrm{Z}$ & GLY/482/O & $\mathrm{TYR} / 32 / \mathrm{OH}$ & 3.0 & Multivalency transforms antibodies into broad and ultrapotent neutralizers [25] \\
\hline $7 \mathrm{~K} 8 \mathrm{~V}$ & $\mathrm{THR} / 345 / \mathrm{O}$ & TYR/31/CE1 & 3.0 & " neutralizing antibody structures inform therapeutic strategies" [26]. \\
\hline 7JMP & $\mathrm{GLU} / 484 / \mathrm{O}$ & $\mathrm{TYR} / 58 / \mathrm{OH}$ & 2.5 & An alternative binding mode of IGHV3-53 antibodies to the RBD [27] \\
\hline $7 \mathrm{CWO}$ & THR/470/O & $\mathrm{ASP} / 54 / \mathrm{OD} 2$ & 3.0 & "Rational development of a human antibody cocktail that deploys multiple functions.." [28] \\
\hline 7CDJ & TYR/449/OH & ARG/72/O & 2.4 & not published \\
\hline $7 \mathrm{C} 8 \mathrm{~W}$ & CYS/488/O & $\mathrm{ARG} / 59 / \mathrm{NH} 2$ & 2.4 & not published \\
\hline 7L02 & SER/940/OG & ARG/99/NH2 & 3.0 & A glycan cluster on the ectodomain recognized by Fab-dimerized glycan-reactive antibodies. [29] \\
\hline
\end{tabular}


Table 2: Residues in SARS-Cov2 protein involved in binding to the ACE2 receptor The reason for the 501Y.V2 variant found in UK and South Africa can be seen - this residue is involved in ACE2 binding.

\begin{tabular}{|c|c|c|c|}
\hline PDB & ACE2 & Cov2 spike & Dist \\
\hline \multirow{19}{*}{ 7KMS [30] } & TYR/41/OH & THR/500/OG1 & 2.2 \\
\hline & GLN/42/OE1 & GLN/498/NE2 & 2.9 \\
\hline & LYS/353/O & GLY/502/N & 3.1 \\
\hline & $\mathrm{PHE} / 28 / \mathrm{CB}$ & TYR/489/OH & 3.2 \\
\hline & GLY/354/O & GLY/502/CA & 3.3 \\
\hline & $\mathrm{ASP} / 355 / \mathrm{CG}$ & THR/500/O & 3.3 \\
\hline & LYS/353/O & TYR/505/CB & 3.3 \\
\hline & TYR/41/OH & $\mathrm{THR} / 500 / \mathrm{CB}$ & 3.4 \\
\hline & $\mathrm{LYS} / 353 / \mathrm{O}$ & TYR/505/CG & 3.4 \\
\hline & LYS/353/C & TYR/505/CD1 & 3.4 \\
\hline & TYR/41/OH & ASN/501/OD1 & 3.5 \\
\hline & $\mathrm{PHE} / 28 / \mathrm{CA}$ & TYR/489/OH & 3.5 \\
\hline & $\mathrm{ASP} / 355 / \mathrm{OD} 1$ & THR/500/O & 3.5 \\
\hline & TYR/41/CZ & THR/500/OG1 & 3.5 \\
\hline & $\mathrm{ASP} / 355 / \mathrm{OD} 2$ & THR/500/OG1 & 3.5 \\
\hline & $\mathrm{ASP} / 355 / \mathrm{OD} 2$ & THR/500/O & 3.5 \\
\hline & THR/27/CG2 & TYR/489/CD1 & 3.5 \\
\hline & $\mathrm{TYR} / 41 / \mathrm{OH}$ & $\mathrm{THR} / 500 / \mathrm{C}$ & 3.5 \\
\hline & $\mathrm{HIS} / 34 / \mathrm{ND} 1$ & $\mathrm{LEU} / 455 / \mathrm{CD} 2$ & 3.5 \\
\hline \multirow{17}{*}{$\begin{array}{l}\text { 7BH9E } \\
\text { (Y501) }\end{array}$} & TYR/41/OH & THR/500/OG1 & 2.6 \\
\hline & LYS/353/O & GLY/502/N & 2.7 \\
\hline & GLN/24/OE1 & ASN/487/ND2 & 2.9 \\
\hline & $\mathrm{ASP} / 355 / \mathrm{OD} 2$ & THR/500/O & 3.0 \\
\hline & TYR/41/CD2 & ARG/498/NH1 & 3.2 \\
\hline & $\mathrm{TYR} / 41 / \mathrm{OH}$ & THR/500/O & 3.2 \\
\hline & $\mathrm{ASP} / 30 / \mathrm{OD} 1$ & PHE/456/CE1 & 3.3 \\
\hline & TYR/41/CE2 & ARG/498/NH1 & 3.3 \\
\hline & $\mathrm{LYS} / 353 / \mathrm{CD}$ & TYR/501/OH & 3.3 \\
\hline & LYS/353/NZ & TYR/505/CD2 & 3.3 \\
\hline & LYS/353/NZ & TYR/505/CE2 & 3.4 \\
\hline & $\mathrm{LYS} / 353 / \mathrm{CD}$ & TYR/501/CZ & 3.4 \\
\hline & GLY /354/O & GLY/502/CA & 3.4 \\
\hline & SER/19/N & ASN/477/OD1 & 3.4 \\
\hline & $\mathrm{ASP} / 30 / \mathrm{OD} 1$ & PHE/456/CZ & 3.5 \\
\hline & $\mathrm{ASP} / 355 / \mathrm{CG}$ & THR/500/O & 3.5 \\
\hline & LYS/353/O & GLY/502/CA & 3.5 \\
\hline
\end{tabular}

Table 3: Variant of concern: Antibodies binding to those mutations - closest distance chosen. B.1.1.7 (UK), P.1 (Brazil), B.1.351 (SA) https://www.cdc.gov/coronavirus/2019-ncov/cases-updates/variantsurveillance/variant-info.html

\begin{tabular}{|c|c|c|c|c|}
\hline Variant & PDB & Spike & Antibody & $\AA$ \\
\hline P.1, B.1.351 & 7CDJ & GLU/484/OE1 & TYR/33/OH & 2.5 \\
P.1, B.1.351 & 7KFW & LYS/417/NZ & SER/98/O & 2.8 \\
New York variant & 7CJF & SER/477/N & GLY/26/ & 3.1 \\
B.1.1.7 & 6ZER & ASN/501/O & VAL/503/CG2 & 3.4 \\
California variant & 7BWJ & LEU/452/CD1 & VAL/105/CG2 & 3.5 \\
\hline
\end{tabular}

